\title{
Congenital coronary anomalies detected by coronary computed tomography compared to invasive coronary angiography
}

Jelena R Ghadri ${ }^{1,2^{*}{ }^{+}}$, Egle Kazakauskaite ${ }^{2,3+}$, Stefanie Braunschweig ${ }^{1}$, Irene A Burger ${ }^{2}$, Michelle Frank ${ }^{1}$, Michael Fiechter ${ }^{2}$, Catherine Gebhard ${ }^{1}$, Tobias A Fuchs ${ }^{2}$, Christian Templin ${ }^{1}$, Oliver Gaemperli 1,2, Thomas F Lüscher ${ }^{1,4}$, Christian Schmied ${ }^{1 \dagger}$ and Philipp A Kaufmann ${ }^{2,4 \dagger}$

\begin{abstract}
Background: As coronary computed tomography angiography (CCTA) has emerged as a non-invasive alternative for evaluation of coronary anatomy with a lower referral threshold than invasive coronary angiography (ICA), the prevalence of coronary anomalies in CCTA may more closely reflect the true prevalence in the general population. Morphological features of coronary anomalies can be evaluated more precisely by CCTA than by ICA, which might lead to a higher identification of congenital coronary anomalies in CCTA compared to ICA.

To evaluate the incidence, clinical and morphological features of the anatomy of patients with coronary anomalies detected either by coronary computed tomography angiography (CCTA) with prospective ECG-triggering or invasive coronary angiography (ICA).
\end{abstract}

Methods: Consecutive patients underwent 64-slice CCTA $\left(n=1^{\prime} 759\right)$ with prospective ECG-triggering or ICA $\left(n=9^{\prime}\right.$ 782) and coronary anatomy was evaluated for identification of coronary anomalies to predefined criteria (origin, course and termination) according to international recommendations.

Results: The prevalence of coronary anomalies was 7.9\% $(n=138)$ in CCTA and $2.1 \%$ in ICA $(n=203 ; p<0.01)$. The most commonly coronary anomaly detected by CCTA was myocardial bridging $42.8 \%$ ( $n=59)$ vs. $21.2 \%$ $(n=43) ; p<0.01$, while with ICA an absent left main trunk was the most observed anomaly $36.0 \%(n=73 ; p<0.01)$. In $9.4 \%(n=13)$ of identified coronary anomalies in CCTA 9.4\% were potentially serious coronary anaomalies, defined as a course of the coronary artery between aorta and pulmonary artery were identified.

Conclusion: The prevalence of coronary anomalies is substantially higher with CCTA than ICA even after exclusion of patients with myocardial bridging which is more frequently found with CCTA. This suggests that the true prevalence of coronary anomalies in the general population may have been underestimated based on ICA.

Keywords: Coronary anomalies, Computed coronary tomography angiography, Invasive coronary angiography

\section{Background}

Coronary anomalies are defined as morphological features occurring in less than $1 \%[1,2]$ of an unselected population affecting the origin, course or termination of a coronary vessel [3]. Based on extrapolations of findings obtained during invasive coronary angiography (ICA) using this

\footnotetext{
* Correspondence: jelena-rima.ghadri@usz.ch

${ }^{\dagger}$ Equal contributors

'Department of Cardiology, University Hospital Zurich, Zurich, Switzerland 2Departement of Nuclear Medicine, Cardiac Imaging University Hospital Zurich, Ramistrasse 100, NUK C 40, Zurich CH-8091, Switzerland

Full list of author information is available at the end of the article
}

definition, the prevalence of any kind of coronary anomaly has been estimated at about $5.6 \%$ for the general population [3]. Better knowledge and early detection of coronary anomalies seems pertinent in view of the fact that they represent the underlying disease in approximately 19\% of sudden cardiac death (SCD) in young athletes [4]. So far, ICA has been considered the first-line method for the assessment of coronary anomalies. Most commonly, coronary anomalies have been detected incidentally during the evaluation of patients with suspected coronary artery disease (CAD). In recent years, coronary computed 
tomography angiography (CCTA) has emerged as a noninvasive alternative for evaluation of coronary anatomy [5], particularly after introduction of modern protocols [6-8] allowing to perform CCTA with a radiation dose substantially lower than that of ICA [9]. CCTA has therefore been recommended as first-line method for the assessment of known or suspected coronary anomalies [10]. The availability of low dose scan protocols has lowered the threshold for referrals to CCTA and is gradually changing the evaluation strategy in many patients with low pre-test probability for CAD. Thus, the prevalence of coronary anomalies in CCTA may more closely reflect their true prevalence in the general population [11].

The aim of the present study was to compare the prevalence and anatomical characteristics of coronary anomalies in a large consecutive population undergoing either CCTA or ICA for different indications.

\section{Methods}

\section{Selection of subjects}

The prevalence of coronary anomalies in both diagnostic approaches (ICA and CCTA) was assessed retrospectively from the CCTA and angiography registry at the University Hospital Zurich. ICA or CCTA were performed due to suspicion of coronary anomaly or coronary heart disease as substantiated by symptoms such as angina pectoris or dyspnea.

We included all consecutive patients undergoing CCTA from February 2007 to October 2011 or ICA from August 2005 to July 2009. The reports of these patients were reviewed for coronary anomalies according to the definition suggested by Angelini et al. [3]. Cardiovascular risk factors and symptoms were obtained from medical records from our hospital based software system. The need for written informed consent was waived by the institutional review board (local ethics committee) due to the retrospective nature of the study with sole clinical data collection.

\section{CT data acquisition and post-processing}

All scans were performed on a 64-slice CT scanner (LightSpeed VCT, GE Healthcare) with prospective ECGtriggering allowing acquisition of low dose CCTA as previously reported [7].

Briefly, intravenous metoprolol (5 to $20 \mathrm{mg}$ ) (Beloc, AstraZeneca, London, UK) was administered to achieve a target heart rate below 65 b.p.m before scanning. A single dose of $2.5 \mathrm{mg}$ isosorbiddinitrate sublingual (Isoket, Schwarz Pharma, Monheim, Germany) was also applied prior to the scan. All patients were carefully monitored during the examination to assure that breathing commands were adequately followed [12]. All images were transferred to an external workstation (AW 4.4, GE Healthcare) for image reconstruction and evaluation.

In all patients in which a coronary anomaly, origin, course and/or termination of the coronary arteries had been originally reported, the angiograms were reassessed by two physicians experienced in cardiac computed tomography imaging.

\section{Invasive coronary angiography}

ICA was performed according to standard techniques on an Allura 9 and an Allura XPER FD10/10 (Philips Medical Systems) catheterization system by experienced interventional cardiologists. Two experienced observers evaluated the coronary arteries based on the results of the invasive assessments.

\section{Effective radiation dose estimation}

Values for radiation dose were estimated for CCTA as the product of the dose length product $(\mathrm{DLP}) \times$ a conversion coefficient for the chest $(\mathrm{k}=0.014 \mathrm{mSv} / \mathrm{mGy} \times \mathrm{cm})$ as previously suggested $[13,14]$. Similarly, for ICA radiation dose was estimated as the product of the dose-area product (DAP) of the diagnostic coronary scenes $x$ conversion factor for chest $\left(\mathrm{k}=0.22\left(\mathrm{mSv} / \mathrm{mGy} \times \mathrm{cm}^{2}\right)\right.$ for ICA based on the National Radiological Protection Board tables [15].

\section{Statistical analysis}

Categorical variables were presented with absolute and relative frequencies (\%), and continuous variables with mean \pm SD. For between group comparisons, unpaired $t$ test were used for parametric data, and Mann-Whitney tests for nonparametric data. A Person $\mathrm{X}^{2}$ or Fisher exact test was performed where appropriate. A $P$ value $<0.05$ was considered to indicate a significant difference. All statistical data were analysed with SPSS software (version 19.0, SPSS Inc., Chicago, Illinois).

\section{Results}

\section{Study population}

From February 2007 to October 2011 CCTA was performed in 1'759 patients revealing 138 coronary anomalies, resulting in a prevalence of $7.9 \%$. From August 2005 to July 2009 ICA was performed in $9^{\prime} 732$ patients and 203 anomalies were found, corresponding to a prevalence of $2.1 \%$.

Patients with coronary anomalies identified with coronary angiography were older compared to those in whom such findings were detected by CCTA. Coronary anomalies tended to occur slightly more often in male $(64.5 \%$ by CCTA and $70.0 \%$ by ICA; $\mathrm{p}=$ n.s.) than in female patients (Table 1). Among the different risk factors, hypertension was most often observed in both patient groups (CT $39.3 \%$ and ICA $62.1 \%(\mathrm{p}<0.01)$; Table 1$)$. Analysis of the symptoms of both groups revealed that typical chest pain 
Table 1 Patient characteristics

\begin{tabular}{|c|c|c|c|}
\hline & CCTA $(n=1759)$ & ICA $(n=9782)$ & $P$ \\
\hline \multicolumn{4}{|l|}{ Patients with coronary anomaly } \\
\hline Total number & 138 & 203 & \\
\hline Prevalence & $7.85 \%$ & $2.08 \%$ & $<0.01$ \\
\hline Age in years (mean $\pm S D$ ) & $56.4 \pm 14.1$ & $68.6 \pm 12.7$ & $<0.01$ \\
\hline Male gender & $89(64.5 \%)$ & $142(70.0 \%)$ & 0.35 \\
\hline \multicolumn{4}{|l|}{ Coronary risk factors } \\
\hline Smoking & $38(27.5 \%)$ & $55(27.1 \%)$ & 0.93 \\
\hline Hypertension & $55(39.3 \%)$ & $126(62.1 \%)$ & $<0.01$ \\
\hline Diabetes & $12(8.7 \%)$ & $33(16.3 \%)$ & 0.06 \\
\hline Positive family history & $38(27.5 \%)$ & $47(23.2 \%)$ & 0.43 \\
\hline Dyslipidemia & $46(32.9 \%)$ & $83(40.9 \%)$ & 0.19 \\
\hline \multicolumn{4}{|l|}{ Clinical symptoms } \\
\hline None & $66(47.8 \%)$ & $31(15.3 \%)$ & $<0.01$ \\
\hline Typical angina & $31(22.5 \%)$ & $106(52.2 \%)$ & $<0.01$ \\
\hline Atypical chest pain & $24(17.1 \%)$ & $41(20.2 \%)$ & 0.61 \\
\hline Dyspnoea & $12(8.6 \%)$ & $56(27.6 \%)$ & $<0.01$ \\
\hline \multicolumn{4}{|l|}{ MPI findings } \\
\hline MPI & $47(34.1 \%)$ & $12(5.9 \%)$ & $<0.01$ \\
\hline Ischemia due to coronary anomaly & 1 & 0 & 0.85 \\
\hline Fixed perfusion defect & 0 & 1 & 0.41 \\
\hline Ischemia due to CAD & 13 & 6 & 0.02 \\
\hline
\end{tabular}

$\mathrm{CCTA}=$ coronary computed tomography angiography, $\mathrm{IAC}=$ invasive coronary angiography, $\mathrm{BMI}=$ body mass index, $\mathrm{HR}=$ heart rate, $\mathrm{MPI}=$ myocardial perfusion imaging, $C A D=$ coronary artery disease.

(52.2\%; $\mathrm{p}<0.01)$ was more common in ICA group, while $47.8 \%$ of patients with anomalies from CCTA demonstrated no symptoms $(\mathrm{p}<0.01)$.

\section{Coronary anomalies}

All anomalies were classified into three main groups: [1] anomalies of origin and course, with a prevalence of $47.8 \%(\mathrm{n}=66$; CCTA $)$ and $64.5 \%(\mathrm{n}=131$; ICA; $\mathrm{p}<0.01)$, respectively. [2] Anomalies of intrinsic coronary arterial anatomy were found in $55.1 \%(\mathrm{n}=76)$ of the patients by CCTA, but only in $26.6 \%(\mathrm{n}=54$ by ICA; $\mathrm{p}<0.01)$. [3] Anomalies of coronary termination were detected in $4.3 \%$ $(n=6)$ of the patients by CCTA and in $8.9 \%(n=18)$ by ICA (Table 2 and Figure 1; $\mathrm{p}=$ n.s.).

\section{Anomalies of origin and course}

In the ICA group, an absent left main trunk was the most frequently observed anomaly occurring in $36.0 \%$ $(\mathrm{n}=73)$ of the patients, while by CCTA such a finding occurred in only $11.6 \%(n=16) ; p<0.01$. In the CCTA group, LCX arising from the right anterior sinus, with anomalous retroaortic course (Figure 2) was noted most commonly $(13.8 \% ; n=19)$, while in the ICA group this anomaly was a rare finding $(2.5 \% ; \mathrm{n}=5 ; \mathrm{p}<0.01)$.
A potentially serious or malignant coronary anomaly was defined as a course of the coronary artery between aorta and pulmonary artery [16]. Obviously, this could only be detected by CCTA imaging (ICA non-accessible; Figure 3). A potentially serious coronary anomaly was found in $9.4 \%(n=13)$ of the patients by CCTA including $8.0 \%(n=11)$ of the patients with a RCA arising from left sinus and $1.5 \%(n=2)$ of the patients with a LAD arising from the opposite sinus, both coursing between the aorta and pulmonary artery. We also included patients with a Bland-White-Garland Syndrome (anomalous origin of the left coronary artery arising from the pulmonary artery) into this group which was detected in $1.5 \%(n=2)$ by CCTA (Figure 4$)$ and in $0.5 \%(n=1)$ by ICA $(p=n . s$. Table 2.

\section{Anomalies of intrinsic coronary arterial anatomy}

The prevalence of an intramural coronary artery (myocardial bridge) was the most common finding with both imaging modalities; however, more cases of myocardial bridging were identified by CCTA $(42.8 \% ; n=59)$ than with ICA $(21.2 \% ; n=43$; $(\mathrm{p}<0.01)$ (Figures 5 and 6$)$. In the CCTA as well as in the ICA group the majority of myocardial bridges were found in the LAD $(n=52 / 59$ 
Table 2 Patients with coronary anomalies

\begin{tabular}{|c|c|c|c|}
\hline & CCTA $(n=138)$ & ICA $(n=203)$ & $P$ \\
\hline 1. Anomalies of origination and course & $66(47.8 \%)$ & $131(64.5 \%)$ & $<0.01$ \\
\hline 1.1 Absent left main trunk (split origination of LMA) & $16(11.6 \%)$ & $73(36.0 \%)$ & $<0.01$ \\
\hline $\begin{array}{l}\text { 1.2 Anomalous location of coronary ostium within aortic root or near proper aortic } \\
\text { sinus of Valsalva }\end{array}$ & $1(0.7 \%)$ & $0(0 \%)$ & 0.84 \\
\hline \multicolumn{4}{|l|}{ 1.3 Anomalous location of coronary ostium outside normal coronary aortic sinuses } \\
\hline LCA arising from posterior facing sinus (ALCAPA) & $2(1.5 \%)$ & $0(0 \%)$ & 0.32 \\
\hline RCA arising from anterior right facing sinus & $3(2.2 \%)$ & $32(15.8 \%)$ & $<0.01$ \\
\hline \multicolumn{4}{|l|}{$\begin{array}{l}\text { 1.4 Anomalous origination of coronary ostium from opposite, facing "coronary" } \\
\text { sinus (which may involve joint origination or adjacent double ostia) }\end{array}$} \\
\hline 1.4.1 RCA arising from left sinus with anomalous course & $11(8.0 \%)$ & $13(6.4 \%)$ & 0.73 \\
\hline Between aorta and pulmonary artery & $11(8.0 \%)$ & n.a. & - \\
\hline 1.4.2 LAD arising from right anterior sinus. with anomalous course & $4(2.9 \%)$ & $7(3.5 \%)$ & 0.78 \\
\hline Between aorta and pulmonary artery & $2(1.5 \%)$ & n.a. & - \\
\hline Anterior to pulmonary outflow or precardiac & $1(0.7 \%)$ & n.a. & - \\
\hline Postero anterior interventricular groove & $1(0.7 \%)$ & n.a. & - \\
\hline 1.4.3 Cx arising from right anterior sinus. with anomalous course & $19(13.8 \%)$ & $5(2.5 \%)$ & $<0.01$ \\
\hline Retroaortic & $19(13.8 \%)$ & n.a. & - \\
\hline 1.5 Single coronary artery & $4(2.9 \%)$ & $0(0 \%)$ & 0.05 \\
\hline 1.6 Bland-White-Garland Syndrome & $2(1.5 \%)$ & $1(0.5 \%)$ & 0.74 \\
\hline 2. Anomalies of intrinsic coronary arterial anatomy; & $76(55.1 \%)$ & $54(26.6 \%)$ & $<0.01$ \\
\hline Absent coronary artery & $4(2.9 \%)$ & n.a. & - \\
\hline Coronary hypoplasia & $11(8.0 \%)$ & n.a. & - \\
\hline Intramural coronary artery (myocardial bridge) & $59(42.8 \%)$ & $43(21.2 \%)$ & $<0.01$ \\
\hline Double RCA & $2(1.5 \%)$ & $2(1.0 \%)$ & 0.70 \\
\hline Double LAD & $0(0 \%)$ & $9(4.4 \%)$ & 0.03 \\
\hline 3. Anomalies of coronary termination, Fistulas from RCA, LCA or infundibular artery to: & $6(4.3 \%)$ & $18(8.9 \%)$ & 0.17 \\
\hline Superior vena cava & $2(1.5 \%)$ & $0(0 \%)$ & 0.32 \\
\hline Pulmonary artery & $1(0.7 \%)$ & $8(3.9 \%)$ & 0.14 \\
\hline Pulmonary vein & $3(2.17 \%)$ & $0(0 \%)$ & 0.13 \\
\hline Right atrium & $0(0 \%)$ & $2(1.0 \%)$ & 0.65 \\
\hline Right ventricle & $0(0 \%)$ & $2(1.0 \%)$ & 0.65 \\
\hline Left atrium & $0(0 \%)$ & $1(0.5 \%)$ & 0.41 \\
\hline Left ventricle & $0(0 \%)$ & $5(2.5 \%)$ & 0.16 \\
\hline
\end{tabular}

$\mathrm{CCTA}=$ coronary computed tomography angiography, IAC=invasive coronary angiography, $\mathrm{LMA}=$ left main coronary artery, $\mathrm{LCA}=$ left coronary artery, $\mathrm{LAD}=$ left anterior descending artery, RCA=right coronary artery, $\mathrm{Cx}=$ circumflex coronary artery.

vs. $\mathrm{n}=42 / 43)$ with the predominant location of the middle segments of the vessel.

\section{Coronary anomalies of termination}

Anomalies of coronary termination were most commonly fistulas originating from the RCA, LCA or infundibular artery (Figure 6). In fact, this group showed the lowest frequency of occurrence. Fistulas either terminated into the pulmonary artery (Figures 7, 8 and 9) in $0.7 \%(n=1)$ of the patients by CTCA and $3.9 \%(n=8)$ by ICA $(\mathrm{p}=\mathrm{n} . \mathrm{s}$. $)$ or into the pulmonary vein in $2.2 \%(\mathrm{n}=3)$ by CTCA, while no patient within the ICA group were identified to have such an anomaly.

\section{Myocardial perfusion imaging}

Myocardial Perfusion Imaging (MPI) was performed in 47 patients $(34.1 \%)$ of the CCTA group and in 12 patients $(5.9 \%)$ of the ICA group $(\mathrm{p}<0.01)$. Despite the high rate of a potentially malignant course of the coronary arteries of $9.4 \%$ in the CTCA group, no ischemia could be attributed to the anomaly itself. However, in one patient out of the entire group of patients with a 


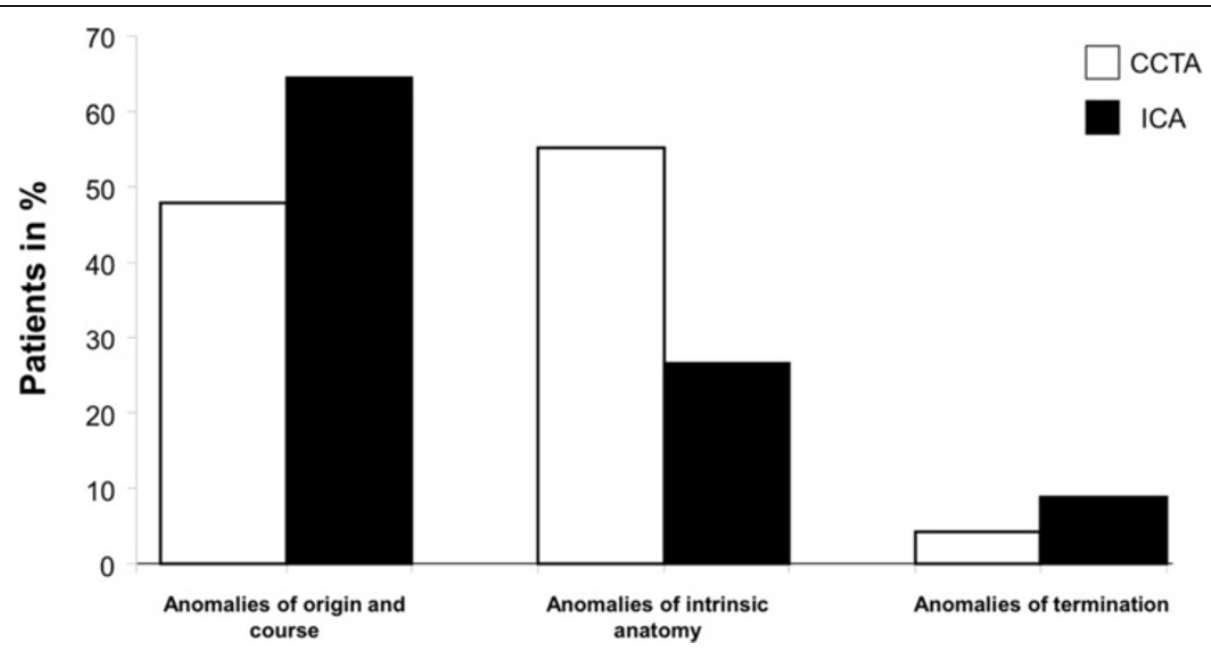

Figure 1 Percentage of patients of the three sub-groups of coronary anomalies identified with both imaging modalities (CCTA/white and ICA/black): 1) Anomalies of origination and course 2) Anomalies of intrinsic coronary artery anatomy 3) Anomalies of coronary termination.

coronary anomaly detected by CCTA myocardial ischemia was noted. No patient of the ICA group that had undergone myocardial perfusion imaging due to coronary anomaly $5.9 \%(\mathrm{n}=12)$ exhibited any perfusion defect (Table 1).

\section{Radiation exposure}

The radiation exposure from CCTA averaged DLP 133.4 \pm $55.6 \mathrm{mGy} \times \mathrm{cm}^{2}$ resulting in $1.9 \pm 0.7 \mathrm{mSv}$. Estimation of the radiation exposure of patients undergoing coronary angiography was limited (due to a separate reporting system). Therefore we assessed the radiation exposure in a random sample of 34 patients out of the 203. The mean DAP was $86.6 \pm 55.4 \mathrm{mGy} \times \mathrm{cm}^{2}$ resulting in a mean radiation dose of $19.1 \pm 12.2 \mathrm{mSv}$.

\section{Invasive procedures in the CCTA group}

Among 138 patients demonstrating a coronary anomaly by CCTA, $22 \%(n=30)$ underwent an additional invasive evaluation and eventually in $12.3 \%(n=17)$ significant coronary artery disease was documented, while the remaining patients $(9.4 \% ; n=13)$ showed no evidence of obstructive coronary artery disease at ICA.

\section{Discussion}

Up until recently, ICA has been considered the gold standard for the evaluation of coronary anomalies.

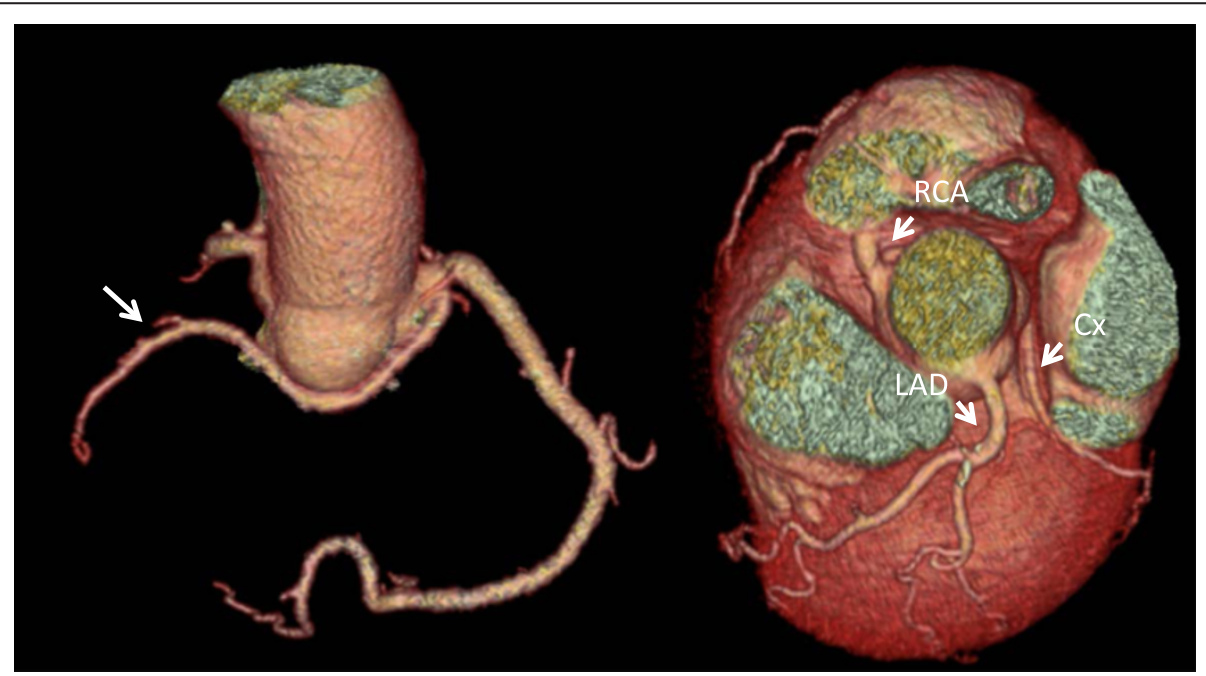

Figure 2 Volume-rendered three-dimensional reconstructions of the coronary tree and heart by CT coronary angiography in a patient presenting with previous syncope showing the circumflex artery arising from the right anterior sinus with anomalous retroaortic course. 


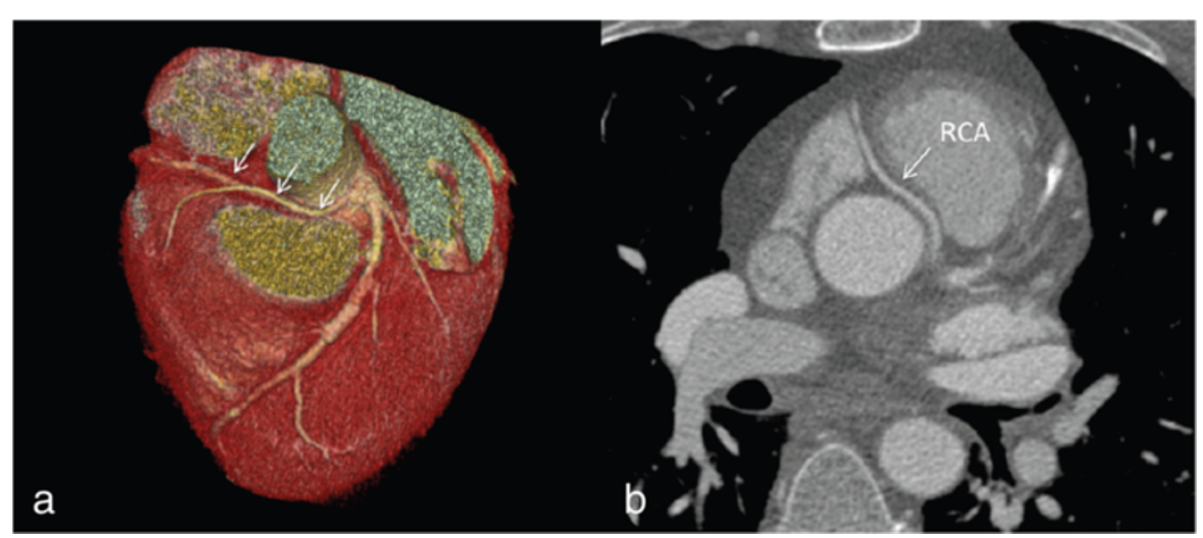

Figure 3 A patient with an anomalous origination of the right coronary artery (RCA). (a) Volume-rendered three-dimensional image showing that RCA is arising from the left coronary sinus with anomalous course between aorta and pulmonary trunk. (b) Axial images of the same patient showing the potentially malignant course of the RCA.

Meanwhile CCTA has emerged as a non-invasive alternative for the evaluation of the coronary architecture, particularly after introduction of modern protocols allowing to perform CCTA with radiation doses substantially lower than that from ICA with improved spatial resolution [17]. We hypothesized that the number of coronary anomalies by CCTA may more closely reflect their real prevalence in the general population. The reported incidences of coronary anomalies were mostly gained by ICA [18], in which the referral threshold is assumedly higher than for CCTA. Indeed, the number of anomalies identified in our survey by CCTA was remarkably higher compared to previous studies $[19,20]$. However, some subgroups of patients underwent CCTA (for example young patients) because of the clinical suspicion of coronary anomalies, while ICA was performed especially for CAD. This issue might partially explain the higher rates of coronary anomalies discovered by CCTA creating a selection bias. Nevertheless the overall prevalence of coronary anomalies detected by CCTA was significantly higher than by coronary angiography (7.85\% versus $2.02 \% ; \mathrm{p}<0.01)$. In this regard, it has to be stated, that the prevalence is also highly dependent on the definition of coronary anomalies. One of the largest clinical data sets which was reported by the Cleveland Clinic Foundation, observed coronary anomalies in $1.3 \%\left(\mathrm{n}=1^{\prime} 461\right)$ out of $126^{\prime} 595$ investigated patients undergoing ICA [21].

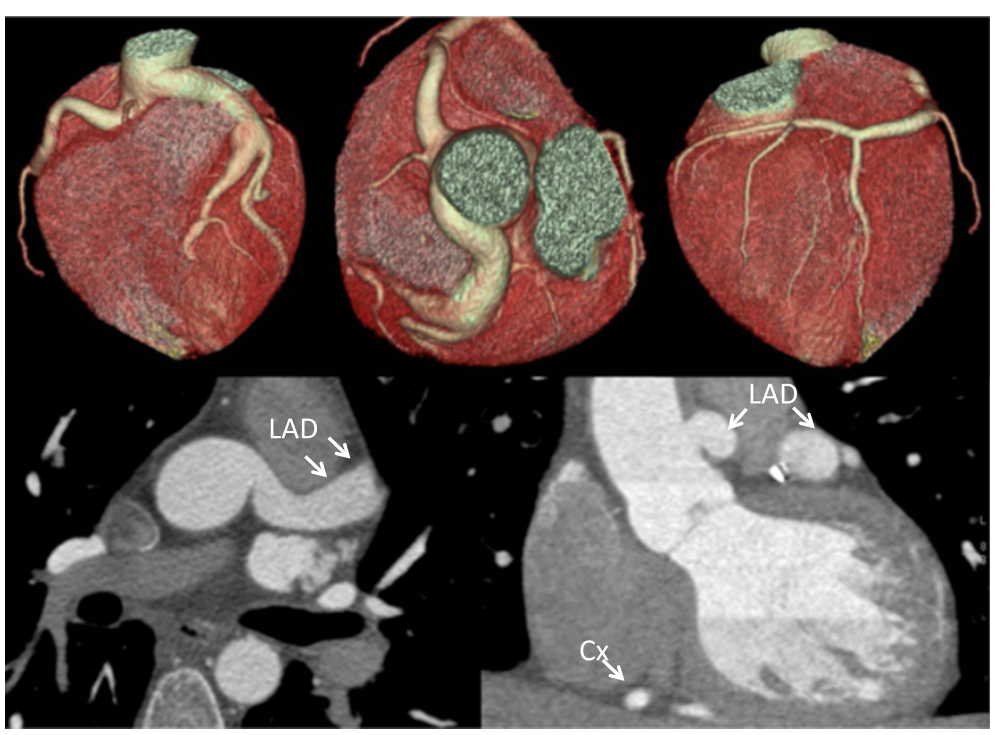

Figure $4 \mathrm{~A}$ patient with bland-white-garland syndrome (anomalous origin of the left coronary artery arising from the pulmonary artery) after cardiac surgery and replacement of origination. Volume-rendered images reveal now, corrected origination of the left coronary artery from the aorta with massive dilatation of the main stem $(14.0 \mathrm{~mm})$ and LAD $(11.4 \mathrm{~mm})$. 


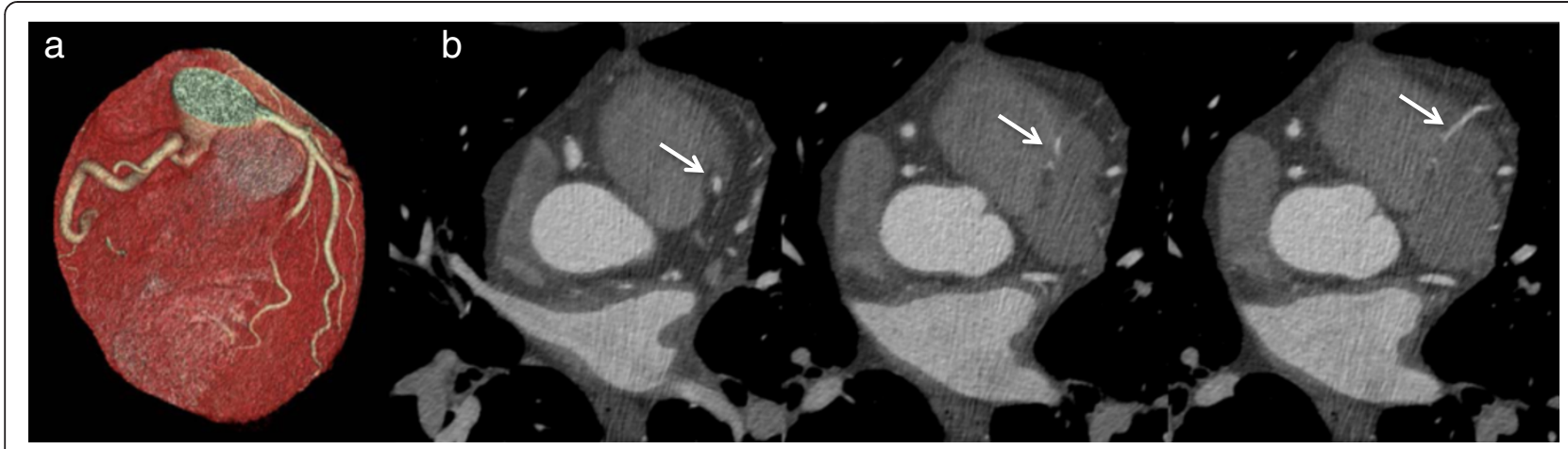

Figure 5 (a) Volume-rendered reconstruction showing myocardial bridge of the left anterior descending artery in a 65-year-old man who was referred for the exclusion of coronary artery disease. (b) Axial images revealing myocardial bridge with intramuscular course of the proximal left anterior descending artery between the left ventricular muscles.

In the present study, coronary anomalies were divided into two groups, i.e. anomalies of the origin/distribution and anomalies of termination. The clinically most relevant coronary anomalies are those with an anomalous course between the large intra-thoracic vessels (aorta and pulmonary artery). In our study, anomalies coursing between the aorta and the pulmonary trunk showed a prevalence of $9.4 \%$ by CCTA, which represents a higher occurrence compared to former studies, primarily based on autopsy data $[22,23]$. Of note, a discrimination of the course of a potentially malignant anomaly is only of reliable accuracy by CCTA, while ICA is limited by its 2dimensional projections. Notably, none of our patients with an abnormal course revealed any perfusion defects by myocardial perfusion imaging. At our institution stress testing is usually performed with adenosine unless contraindicated. In this regard some of our patients $(n=5 / 13)$ with potentially serious coronary anomalies in the CCTA group were stressed with adenosine, although exercise testing is more physiological as the coronary artery may be squeezed between the pulmonary artery trunc and the aorta. However, these patients were usually referred for a combined investigation with CCTA and SPECT, and incidentally diagnosed with a potentially serious coronary anomaly after the SPECT results. Several studies have reported an increased risk for sudden cardiac death [24] as such patients showing myocardial ischemia [25]. However, only limited data are available concerning the long-term outcome of such patients without demonstrable myocardial ischemia $[3,26]$. Certainly, asymptomatic patients without stress-induced ischemia remain a clinical challenge. There is still controversy concerning the management of these patients. While some favor a surgical approach others recommend a conservative management. Since sudden cardiac death is associated with this anomaly related to severe exercise, patients are recommended to avoid extreme exertion and should be followed medically.

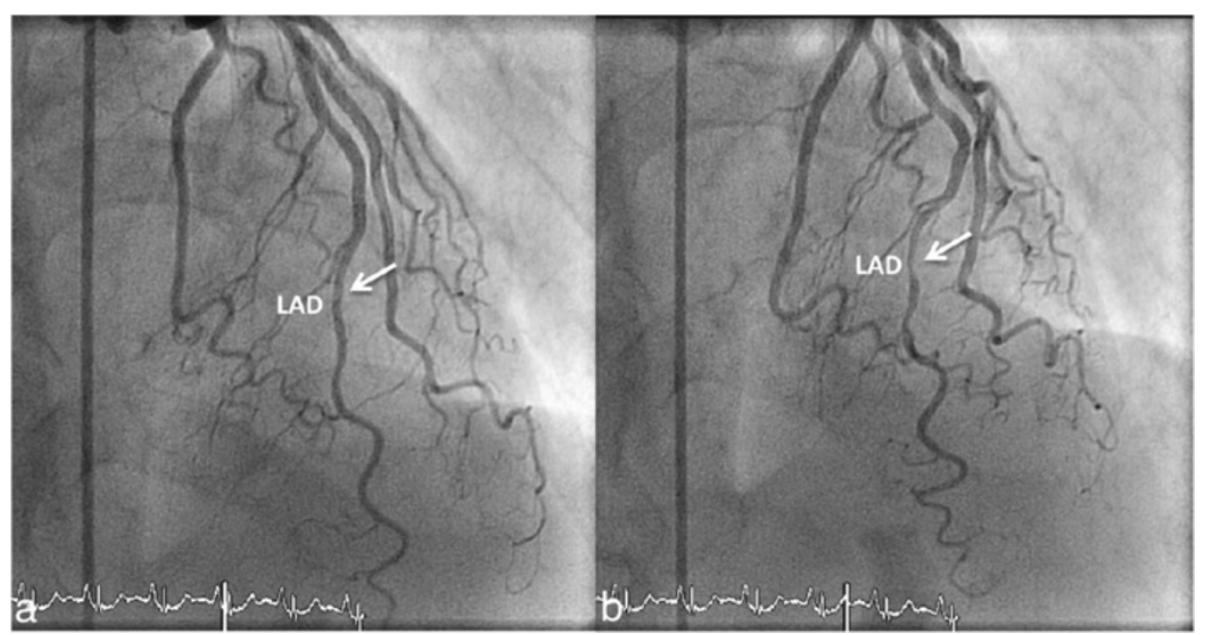

Figure 6 A patient with angina pectoris underwent coronary angiography. Images reveal myocardial bridging in the mid LAD (a) during diastole and (b) during systole. 


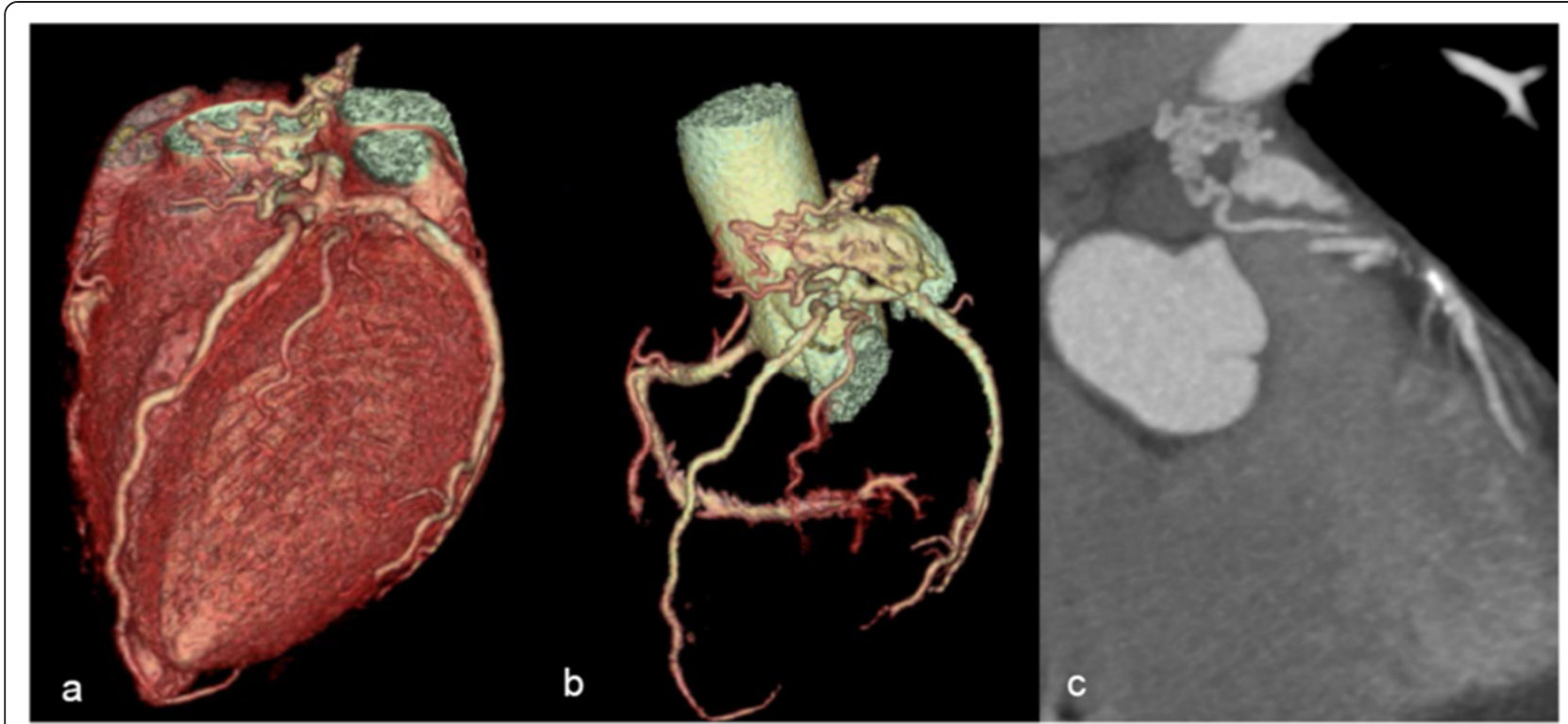

Figure $7 \mathrm{~A}$ patient with a complex coronary-pulmonary artery fistula presenting with previous syncope. $(\mathbf{a}, \mathbf{b})$ Volume-rendered three-dimensional images show a big plexus of the tortuous vessels arising from the proximal left anterior descending artery. (c) Axial images of the same patient show the course of the fistula ending into a pulmonary vein.

Beside the potential to describe the exact course of aberrant coronary vessels [27], CCTA offers another important advantage: Radiation dose exposure can be reduced to very low levels by applying novel CT protocols such as prospective ECG-triggering [6-9,28], tube current modulation [29] or high-pitch modus [7]. In addition several studies have demonstrated superiority of CCTA over ICA [30] in detecting coronary anomalies leading to a $100 \%$ identification rate of anomalies when analyzed with CCTA compared to 53\% with ICA in a small series of 16 patients [31]. CCTA has recently been advocated as first-line method for known or suspected anomalies by the American College of Cardiology, provided appropriate criteria are used [32].

Interestingly, the most common finding in the CCTA group was myocardial bridging occurring in 43\%, while such an anomaly was found in only $21 \%$ by ICA. Previous studies have reported myocardial bridging in 1.5-16\% when assessed by ICA, while a substantially higher number up to $80 \%$ was observed in autopsy series [33]. Thus, myocardial bridging is a very common finding at autopsy even in normal subjects, but is considered clinically significant only when associated with chest pain and myocardial ischemia $[34,35]$. In our CCTA patient population,

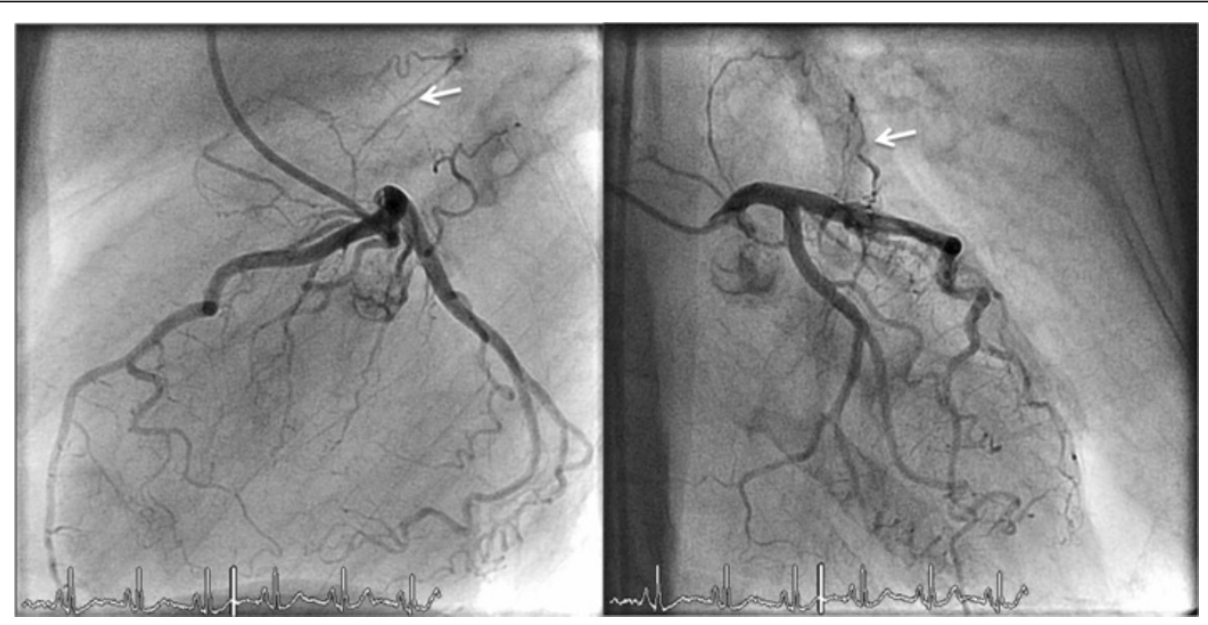

Figure $8 \mathrm{~A}$ patient with angina symptoms during exercise stress test revealed a rudimentary fistula from the LAD to the pulmonary artery. 


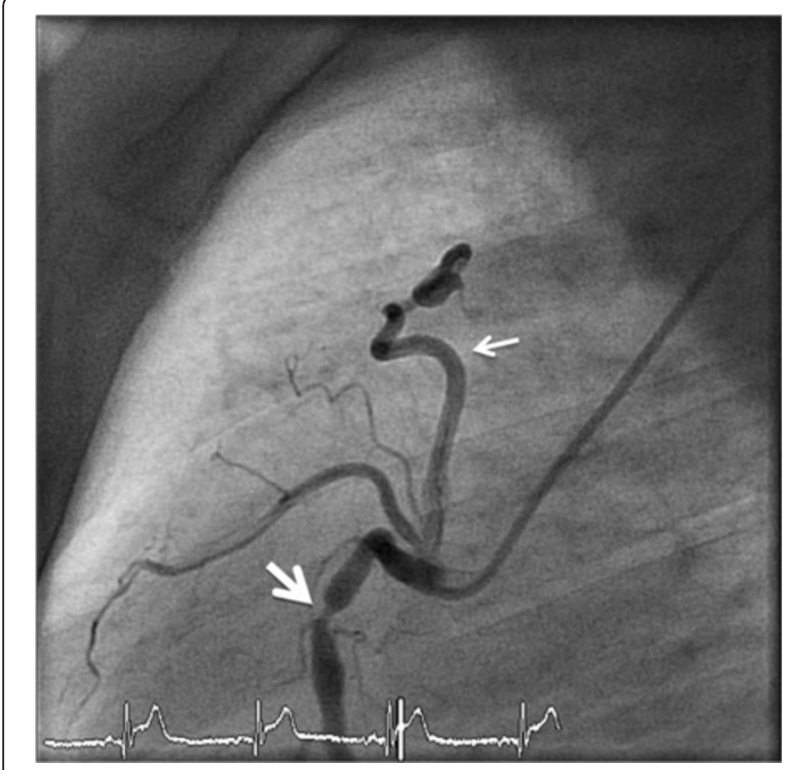

Figure $9 \mathrm{~A}$ patient underwent coronary angiography due to inferior ST-segment elevation myocardial infarction. Coronary images revealed AV-Fistula from the proximal RCA to the pulmonary artery. The (bold arrow) shows the culprit lesion in the mid RCA.

none of the patients with myocardial bridging revealed any perfusion defect in myocardial perfusion studies.

A further very common finding with both imaging modalities was the absence of the left main artery ranging between $12 \%$ by CCTA to $36 \%$ by ICA. These results are in line with Yamanaka et al. who found this anomaly to be most common (30.4\%) among 126'595 patients evaluated by ICA. The prevalence among the general population is considered to be $0.41 \%$ [21]. This anomaly is usually regarded as benign, since the distribution of the vessels is normal.

This was a retrospective analysis and not a head to head comparison between two different techniques. Indeed, only a few patients underwent both imaging modalities. Furthermore this study did not include a randomly assigned study population rather than patients who were primarily referred by cardiologists for evaluation of suspected CAD or coronary anomaly which could have attributed to the higher identification of coronary anomalies among CCTA. Finally, young patients are more likely to be referred to a non-invasive imaging study with CCTA than for ICA, which may have introduced a potential selection bias.

\section{Conclusion}

Our study indicates that coronary anomalies can be detected with both imaging modalities; however CCTA is superior in identifying the course of potentially malignant anomalies. The prevalence of coronary anomalies is substantially higher on CCTA than ICA even after exclusion of patients with myocardial bridging which is more frequently found with CCTA. This suggests that the true prevalence of coronary anomalies in the general population may have been underestimated based on ICA.

\section{Abbreviation \\ CAD: Coronary artery disease; CCTA: Coronary computed tomography angiography; CT: Computer tomography; ICA: Intracoronary angiography.}

\section{Competing interests}

The authors declare that they have no competing interest.

\section{Authors' contributions}

All authors have substantially contributed to the submitted work. All authors read and approved the final manuscript.

\section{Author details}

${ }^{1}$ Department of Cardiology, University Hospital Zurich, Zurich, Switzerland ${ }^{2}$ Departement of Nuclear Medicine, Cardiac Imaging University Hospital Zurich, Ramistrasse 100, NUK C 40, Zurich CH-8091, Switzerland. ${ }^{3}$ Hospital of Lithuanian University of Health Sciences Kaunas Clinics, Kaunas, Lithuania. ${ }^{4}$ Zurich Center of Integrative Human Physiology (ZIHP) University of Zurich, Zurich, Switzerland.

\section{Received: 25 September 2013 Accepted: 22 May 2014}

Published: 8 July 2014

\section{References}

1. Baltaxe HA, Wixson D: The incidence of congenital anomalies of the coronary arteries in the adult population. Radiology 1977, 122:47-52.

2. Click RL, Holmes DR Jr, Vlietstra RE, Kosinski AS, Kronmal RA: Anomalous coronary arteries: location, degree of atherosclerosis and effect on survival-a report from the coronary artery surgery study. J Am Coll Cardiol 1989, 13:531-7

3. Angelini P, Velasco JA, Flamm S: Coronary anomalies: incidence, pathophysiology, and clinical relevance. Circulation 2002, 105:2449-54.

4. Maron BJ, Thompson PD, Puffer JC, McGrew CA, Strong WB, Douglas PS, Clark LT, Mitten MJ, Crawford MH, Atkins DL, Driscoll DJ, Epstein AE: Cardiovascular preparticipation screening of competitive athletes: a statement for health professionals from the sudden death committee (clinical cardiology) and congenital cardiac defects committee (cardiovascular disease in the young), American heart association. Circulation 1996, 94:850-6.

5. Dhoble A, Dewar J, Szerlip M, Abidov A: Rare coronary anomaly: Common origin of major coronary arteries from the right sinus of Valsalva and a small ramus branch originating from the left sinus of Valsalva. Int $\mathrm{J}$ Cardiol 2011.

6. Husmann L, Herzog BA, Gaemperli O, Tatsugami F, Burkhard N, Valenta I, Veit-Haibach P, Wyss CA, Landmesser U, Kaufmann PA: Diagnostic accuracy of computed tomography coronary angiography and evaluation of stress-only single-photon emission computed tomography/computed tomography hybrid imaging: comparison of prospective electrocardiogram-triggering vs. retrospective gating. Eur Heart J 2009, 30:600-7.

7. Ghadri JR, Kuest SM, Goetti R, Fiechter M, Pazhenkottil AP, Nkoulou RN, Kuhn FP, Pietsch C, von Schulthess P, Gaemperli O, Templin C, Kaufmann $P A$ : Image quality and radiation dose comparison of prospectively triggered low-dose CCTA: 128-slice dual-source high-pitch spiral versus 64-slice single-source sequential acquisition. Int J Cardiovasc Imaging 2011.

8. Achenbach S, Marwan M, Ropers D, Schepis T, Pflederer T, Anders K, Kuettner A, Daniel WG, Uder M, Lell MM: Coronary computed tomography angiography with a consistent dose below $1 \mathrm{mSv}$ using prospectively electrocardiogram-triggered high-pitch spiral acquisition. Eur Heart $J$ 2010, 31:340-346.

9. Herzog BA, Wyss CA, Husmann L, Gaemperli O, Valenta I, Treyer V, Landmesser U, Kaufmann PA: First head-to-head comparison of effective radiation dose from low-dose 64-slice CT with prospective ECGtriggering versus invasive coronary angiography. Heart 2009, 95:1656-61. 
10. Schroeder $S$, Achenbach $S$, Bengel $F$, Burgstahler $C$, Cademartiri $F$, de Feyter P, George R, Kaufmann P, Kopp AF, Knuuti J, Ropers D, Schuijf J, Tops LF, Bax JJ: Cardiac computed tomography: indications, applications, limitations, and training requirements: report of a writing group deployed by the working group nuclear cardiology and cardiac CT of the European society of cardiology and the European council of nuclear cardiology. Eur Heart J 2008, 29:531-56.

11. Villines TC, Devine PJ, Cheezum MK, Gibbs B, Feuerstein IM, Welch TS: Incidence of anomalous coronary artery origins in 577 consecutive adults undergoing cardiac CT angiography. Int J Cardiol 2010, 145:525-6.

12. Husmann L, Herzog BA, Pazhenkottil AP, Buechel RR, Nkoulou R, Ghadri JR, Valenta I, Burger IA, Gaemperli O, Wyss CA, Kaufmann PA: Lowering heart rate with an optimised breathing protocol for prospectively ECGtriggered CT coronary angiography. Br J Radiol 2011, 84:790-5.

13. Einstein AJ, Moser KW, Thompson RC, Cerqueira MD, Henzlova MJ: Radiation dose to patients from cardiac diagnostic imaging. Circulation 2007, 116:1290-305.

14. Hausleiter J, Meyer T, Hermann F, Hadamitzky M, Krebs M, Gerber TC, McCollough C, Martinoff S, Kastrati A, Schomig A, Achenbach S: Estimated radiation dose associated with cardiac CT angiography. JAMA 2009, 301:500-7.

15. Lobotessi H, Karoussou A, Neofotistou V, Louisi A, Tsapaki V: Effective dose to a patient undergoing coronary angiography. Radiat Prot Dosimetry 2001, 94:173-6.

16. Basso C, Maron BJ, Corrado D, Thiene G: Clinical profile of congenital coronary artery anomalies with origin from the wrong aortic sinus leading to sudden death in young competitive athletes. J Am Coll Cardiol 2000, 35:1493-501.

17. Gaudio C, Nguyen BL, Tanzilli G, Mirabelli F, Catalano C: Anomalous "benign" coronary arteries detected by multidetector computed tomography. Int J Cardiol 2006, 109:417-9.

18. Garg N, Tewari S, Kapoor A, Gupta DK, Sinha N: Primary congenital anomalies of the coronary arteries: a coronary: arteriographic study. Int J Cardiol 2000, 74:39-46.

19. Tariq R, Kureshi SB, Siddiqui UT, Ahmed R: Congenital anomalies of coronary arteries: Diagnosis with 64 slice multidetector CT. Eur J Radiol 2011.

20. Zhang LJ, Yang GF, Huang W, Zhou CS, Chen P, Lu GM: Incidence of anomalous origin of coronary artery in 1879 Chinese adults on dual-source CT angiography. Neth Hear J 2010, 18:466-470.

21. Yamanaka O, Hobbs RE: Coronary artery anomalies in 126,595 patients undergoing coronary arteriography. Cathet Cardiovasc Diagn 1990, 21:28-40.

22. Frescura C, Basso C, Thiene G, Corrado D, Pennelli T, Angelini A, Daliento L: Anomalous origin of coronary arteries and risk of sudden death: a study based on an autopsy population of congenital heart disease. Hum Pathol 1998, 29:689-95.

23. Alexander RW, Griffith GC: Anomalies of the coronary arteries and their clinical significance. Circulation 1956, 14:800-5.

24. Mirchandani S, Phoon CK: Management of anomalous coronary arteries from the contralateral sinus. Int J Cardio/ 2005, 102:383-9.

25. Angelini P: Coronary artery anomalies: an entity in search of an identity. Circulation 2007, 115:1296-305.

26. Uebleis C, Groebner M, von Ziegler F, Becker A, Rischpler C, Tegtmeyer R, Becker C, Lehner S, Haug AR, Cumming P, Bartenstein P, Franz WM, Hacker $\mathrm{M}$ : Combined anatomical and functional imaging using coronary $\mathrm{CT}$ angiography and myocardial perfusion SPECT in symptomatic adults with abnormal origin of a coronary artery. Int J Cardiovasc Imaging 2011.

27. Schmid M, Achenbach S, Ludwig J, Baum U, Anders K, Pohle K, Daniel WG, Ropers D: Visualization of coronary artery anomalies by contrastenhanced multi-detector row spiral computed tomography. Int J Cardiol 2006, 111:430-5.

28. Buechel RR, Husmann L, Herzog BA, Pazhenkottil AP, Nkoulou R, Ghadri JR, Treyer $V$, von Schulthess P, Kaufmann PA: Low-dose computed tomography coronary angiography with prospective electrocardiogram triggering: feasibility in a large population. J Am Coll Cardiol 2011, 57:332-6.

29. Husmann L, Valenta I, Gaemperli O, Adda O, Treyer V, Wyss CA, Veit-Haibach P, Tatsugami F, von Schulthess GK, Kaufmann PA: Feasibility of low-dose coronary $\mathrm{CT}$ angiography: first experience with prospective ECG-gating. Eur Heart J 2008, 29:191-7.
30. Andreini D, Mushtaq S, Pontone G, Cortinovis S, Annoni A, Formenti A, Agostoni P, Bartorelli AL, Fiorentini C, Ballerini G, Pepi M: Additional clinical role of 64-slice multidetector computed tomography in the evaluation of coronary artery variants and anomalies. Int J Cardio/ 2010, 145:388-90.

31. Shi H, Aschoff AJ, Brambs HJ, Hoffmann MH: Multislice CT imaging of anomalous coronary arteries. Eur Radio/ 2004, 14:2172-81.

32. Hendel RC, Patel MR, Kramer CM, Poon M, Carr JC, Gerstad NA, Gillam LD, Hodgson JM, Kim RJ, Lesser JR, Martin ET, Messer JV, Redberg RF, Rubin GD, Rumsfeld JS, Taylor AJ, Weigold WG, Woodard PK, Brindis RG, Douglas PS, Peterson ED, Wolk MJ, Allen JM: ACCF/ACR/SCCT/SCMR/ASNC/NASCI/SCAI/ SIR 2006 appropriateness criteria for cardiac computed tomography and cardiac magnetic resonance imaging: a report of the American college of cardiology foundation quality strategic directions committee appropriateness criteria working group, American college of radiology, society of cardiovascular computed tomography, society for cardiovascular magnetic resonance, American society of nuclear cardiology, North American society for cardiac imaging, society for cardiovascular angiography and interventions, and society of interventional radiology. J Am Coll Cardiol 2006, 48:1475-97.

33. Rossi L, Dander B, Nidasio GP, Arbustini E, Paris B, Vassanelli C, Buonanno C, Poppi A: Myocardial bridges and ischemic heart disease. Eur Heart J 1980, $1: 239-45$.

34. Kulan K, Kulan C, Tuncer C, Komsuoglu B, Telatar M: Myocardial perfusion scintigraphy in a myocardial bridging of coronary artery. Clin NuCl Med 1996, 21:888-9.

35. Marcos-Alberca P, Goncalves A, Golfin CF, Ibanez B, Castilla E, Blanco E, Ferreiros J, Arrazola J, Macaya C, Zamorano J: Clinical outcomes of patients with intramyocardial bridging diagnosed by multi-detector cardiac computed tomography. Int J Cardiol 2011, 148:123-5.

doi:10.1186/1471-2261-14-81

Cite this article as: Ghadri et al: Congenital coronary anomalies detected by coronary computed tomography compared to invasive coronary angiography. BMC Cardiovascular Disorders 2014 14:81.

\section{Submit your next manuscript to BioMed Central and take full advantage of:}

- Convenient online submission

- Thorough peer review

- No space constraints or color figure charges

- Immediate publication on acceptance

- Inclusion in PubMed, CAS, Scopus and Google Scholar

- Research which is freely available for redistribution

Submit your manuscript at www.biomedcentral.com/submit
C) Biomed Central 